\title{
On-site comparison of an enzymatic detergent and a non-enzymatic detergent-disinfectant for routine manual cleaning of flexible endoscopes
}

\section{(ㄷ)(1) $(-)$}

\author{
Authors
}

Jonathan Alfageme Gonzalez ${ }^{*}{ }^{1}$, Thomas Vanzieleghem ${ }^{*}, 2$, Axelle Dumazy ${ }^{1}$, Christelle Meuris ${ }^{1}$, Jacques Mutsers ${ }^{1}$, Genevieve Christiaens ${ }^{1}$, Philippe Leclercq ${ }^{3}$, Jean-Philippe Loly ${ }^{3}$, Edouard Louis ${ }^{1}$, Pierrette Gast ${ }^{3}$ in the outcome of the manual cleaning of endoscopes. was to demonstrate the impact of the cleaning chemistry

Materials and methods Twelve endoscopes were included in this study: four colonoscopes, four gastroscopes, two duodenoscopes and two bronchoscopes. This study was designed with two phases; in each of them, the manual cleaning procedure remained identical, but a different detergent was used: a non-enzymatic detergent-disinfectant (NEDD) and an enzymatic detergent (ED). Biopsy and suction channels of endoscopes were sampled using $10 \mathrm{~mL}$ of physiological saline at two points: before and after manual cleaning, and adenosine triphosphate (ATP) was measured on each sample. In total, 208 procedures were analyzed for the NEDD phase and 253 for the ED phase.

Results For each endoscope type, cleaning endoscopes with ED resulted in larger median decrease in ATP than with NEDD: respectively $99.43 \%$ and $95.95 \%$ for bronchoscopes $(P=0.0007), 99.28 \%$ and $96.93 \%$ for colonoscopes $(P<0.0001)$ and $98.36 \%$ and $95.36 \%$ for gastroscopes $(P<$ $0.0001)$. In addition, acceptability rates of endoscopes based on defined post-manual cleaning ATP thresholds $(200,150,100$ or 50 relative light units) for all endoscope types were significantly higher with ED compared to NEDD. Conclusions With all other parameters of manual cleaning remaining unchanged, the enzymatic chemistry of ED provided more consistent and improved cleaning of endoscopes compared to NEDD. Therefore, choice of the detergent for endoscope cleaning has an impact on the outcome of this process.

\section{Introduction}

Flexible endoscopes are categorized as semi-critical items [13]. These medical devices, which contact mucous membranes and/or non-intact skin, should be free from all microorganisms (except a small number of bacterial spores, considered as permissible), and therefore minimally require high-level disinfection (HLD) [1].

\footnotetext{
* These authors contributed equally.
}

Evidence-based endoscope reprocessing guidelines and recommendations have been developed and published by numerous professional and public health organizations [1,4-8], providing extensive information about endoscope cleaning and disinfection. However, despite apparent compliance with these guidelines, endoscopes still carrying bioburden after HLD have been found to contribute to pathogen transmission to patients during endoscopic procedures [9]. Some cases of outbreaks, notably involving extended-spectrum beta-lactamase-producing Pseudomonas aeruginosa [10] and carbapenem-resistant 
Enterobacteriaceae [11-12] as main pathogens were also reported to be linked to contaminated endoscopes.

Microbial biofilm is one important factor that allows bacterial persistence in endoscopes despite HLD. In a recent research conducted by Neves and a team of gastroenterologists in 2016 , the authors compared the efficacy of current disinfection methods after manual cleaning on a surrogate endoscope model contaminated with biofilm. They highlighted that, although culturable germs could not be found, very low levels of biofilm persisted in the endoscope model, allowing the persistence of dormant, non-culturable germs. Based on these findings, they suggest that eradicating biofilms from endoscopes is a priority [13]. Of note, this study was focused on disinfection methods and the role of manual cleaning of endoscopes prior to disinfection was not investigated, despite the fact that the results show that the larger reduction of bioburden in endoscopes happened after manual cleaning. Similar conclusions were drawn in other studies [14-16].

There is increased recognition that current reprocessing procedures may not be sufficient to ensure germ-free endoscopes [17-19]. Optimization of manual cleaning of endoscopes, an important step in the reprocessing cycle that precedes and is complementary to HLD, may help to improve the outcome of this process and of the whole reprocessing procedure. Factors that can be assessed include, among others, the human factor and possible automation [20] and methods and devices for brushing and flushing of endoscope channels [21]. Recent validation of rapid techniques such as adenosine triphosphate (ATP) quantification to audit manual cleaning by Alfa and colleagues in 2013 [14, 22] opens perspectives for optimization of endoscope manual cleaning procedures. We used the ATP quantification method to determine whether manual cleaning can be improved based on choice of cleaning chemistry. Two cleaning chemistries were compared in this two-phase study: one enzymatic detergent and one non-enzymatic detergent with biocidal properties. For each phase, a subset of 12 endoscopes was repeatedly sampled before and after manual cleaning (i. e. before proceeding to HLD) in distinct procedures. In total for both phases, over 460 procedures were analyzed.

\section{Materials and methods}

\section{Endoscopes included in this study}

The University Hospital of Liège has 50 endoscopes in service. Twelve endoscopes were selected for this study: four gastroscopes, four colonoscopes, two bronchoscopes and two duodenoscopes. All selected endoscopes were manufactured by Olympus, their reference number and date of entry in service at the hospital are summarized in supplementary information - Table S1.

\section{Reprocessing procedure for flexible endoscopes and staff}

The endoscope reprocessing procedure in place at University Hospital Liège is in accordance with guidelines from the European Society of Gastrointestinal Endoscopy (ESGE) and European Society of Gastroenterology Nurses and Associates
(ESGENA) [5]. After an endoscopic procedure on a patient, endoscopes channels are flushed at bedside with sterile water and the external sheath is mopped with a dry wipe. Briefly the endoscopes are transported to the room dedicated to cleaning and disinfection, where they are cleaned manually (immersion in detergent bath with tap water, flushing all channels with detergent solution, brushing with adapted disposable singleended brushes [De Beukelaer, Belgium]) then they are inserted in an AER (Soluscope Series 4, Soluscope, France) for a cycle comprising the following steps: pre-rinse, cleaning with Soluscope CLN (an alkaline cleaner), rinse, disinfection with Soluscope PAA, a peracetic based chemistry and a final rinse with tap water filtered on a 0.22-um cartridge. Endoscopes are then stored in a horizontal storage DSC8000 drying cabinet (Soluscope, France) for maximum 72 hours before use. All endoscopes, whether they were included in this study or not, were reprocessed following the same procedure. The reprocessing staff is composed of four fully-trained people. One extra staff member was added during the course of this study. During the course of this study, one person quit and was replaced by another person.

\section{Detergents used in this study}

In this work, there were two phases, one for each detergent. Detergents used are commercially available and intended for the manual cleaning of endoscopes. The first detergent is a non-enzymatic detergent-disinfectant (NEDD), a pH-neutral cleaner with claimed bactericidal, mycobactericidal, yeast cidal and virucidal activities. NEDD is a Class IIb medical device according to EC 93/42 European directive and 2017/745 European Medical Device Regulation. The main components of NEDD are $\mathrm{N}, \mathrm{N}$-didécyl-N,N-dimethylammonium carbonate and polyhexamethylene biguanide for antimicrobial active ingredients, polyalkoxylated fatty alcohol and Lauryldimethamine oxide for detergent active ingredient, and other ingredients like fragrance, coloring agent, sequestering and dispersing agent. The second detergent is an enzymatic detergent herein referred to as ED, a pH-neutral, multi-enzymatic detergent including protease, alpha-amylase, cellulase and lipase as enzymes, polyethoxylated alcohols and sulfonic acids as detergent active ingredient and phosphonates as sequestering agent. ED is a Class I medical device according to EC 93/42 European directive and 2017/745 Medical Device Regulation. The first detergent (NEDD) was used during the first 67 working days without interruption then ED was used subsequently for 68 working days. The endoscope reprocessing procedure remained identical for both study phases, except for cleaning chemistry used. Contact time was 5 minutes for both detergents as per manufacturer instructions for use. Water temperature was $35^{\circ} \mathrm{C}$.

\section{Endoscope sampling and ATP measurement of endoscope soil level}

Endoscopes included in this study were sampled randomly, whenever staff had sufficient time to do so, at two different time points in the reprocessing cycle to assess their level of soiling. The first sample was collected before proceeding to man- 
- Table 1 Number of reprocessing cycles analyzed with ATP quantification and total number of cycles performed on the endoscopes included in this study.

\begin{tabular}{|l|c|c|c|c|}
\hline \multirow{2}{*}{ Endoscope type } & \multicolumn{2}{|c|}{ Number of reprocessing procedures analyzed } & \multicolumn{2}{|c|}{ Total number of reprocessing procedures performed } \\
\hline & NEDD phase & ED phase & NEDD phase & ED phase \\
\hline Bronchoscope & 17 & 25 & 234 & 57 \\
\hline Colonoscope & 90 & 92 & 868 & 90 \\
\hline Duodenoscope & 8 & 9 & 107 & 956 \\
\hline Gastroscope & 93 & 127 & 1054 & 1708 \\
\hline All types & 208 & 253 & 2263 & \\
\hline $\begin{array}{l}\text { Data are pooled by endoscope type and presented for each phase: NEDD and ED. } \\
\text { ATP, adenosine triphosphate; ED, enzymatic detergent; NEDD, non-enzymatic detergent-disinfectant }\end{array}$
\end{tabular}

ual cleaning and the second sample was collected just after manual cleaning. Endoscopes were not rinsed with clear water after manual cleaning but the detergent solution was drained from the channels by hanging the endoscopes on a suspension device and samples were only collected when no more liquid was dripping from the endoscope channels. The sampling procedure was performed as follows: $5 \mathrm{~mL}$ of sterile saline solution (Baxter, Belgium) was injected in the biopsy channel from the suction valve port and collected from the distal end of the endoscope in a sterile $50-\mathrm{mL}$ conical tube, subsequently, another volume of $5 \mathrm{~mL}$ of sterile saline solution was injected in the suction channel and collected from the umbilical end of the endoscope in the same tube. The endoscope was then placed in an automated endoscope reprocessor to follow the reprocessing cycle.

ATP measurements were performed on samples with EnSure Luminometer and AquaSnap Total ATP tests (Hygiena, Watford, UK) according to manufacturer instructions immediately after the sampling procedure. Relative light units (RLU) measured with the Hygiena Ensure device range from 0 to 9,999. Samples were kept no longer than 10 minutes at room temperature before analysis. A total of 461 procedures were analyzed with ATP monitoring before and after manual cleaning: 208 for the NEDD phase and 253 for the ED phase.

\section{Data and statistical analyzes}

ATP values measured on endoscope samples were recorded as RLU. Operator name, time and date of measurement and the total number of reprocessing cycles undergone by each endoscope were recorded. Measured initial and final ATP values collected in RLU were transformed in $\log 10$ to make the distribution of data more symmetrical. Percentage of decrease between initial and final ATP values was calculated using the following formula $=\%$ decrease ATP $(R L U)=($ initial ATP $(R L U)-$ final ATP (RLU)) / initial ATP (RLU) * $100 \%$.

JMP (SAS Institute Inc., Marlow, Buckinghamshire, UK) was used to perform all statistical analyses and box and whisker plots. For the statistical analyses, ATP values of individual endoscopes were pooled by endoscope type: bronchoscopes, duodenoscopes, gastroscopes and colonoscopes. Initial and final ATP levels for each phase (detergent) and each endoscope type are represented in box and whisker plots. Descriptive statistics (minimum, maximum, first and third quartile, median and mean) and Kruskal Wallis test for equivalence of distributions were calculated for the percentage of decrease between initial and final ATP levels for both detergents and each endoscope type. Finally, likelihood ratio test for independence were performed to assess whether the detergent has an influence on the final level of ATP (i.e. residual soiling after manual cleaning). This analysis was made for four different RLU thresholds: 50, 100, 150, 200 RLUs.

\section{Results}

This study had two phases, one for each detergent tested. ATP quantification was performed on samples taken from endoscopes before and after manual cleaning in both phases of the study (i.e. initial and final ATP levels). Endoscopes were not rinsed post-manual cleaning, but controls with detergent solution showed no contribution to ATP quantification nor did they quench ATP signal (data not shown). > Table 1 summarizes the total number of reprocessing procedures performed and the number of procedures analyzed with ATP measurement for each endoscope type in each of the phases of this study. The absolute number of procedures analyzed for bronchoscopes and duodenoscopes was much lower than for colonoscopes and gastroscopes due to reduced clinical usage of these endoscopes. The proportion of procedures analyzed compared to the total number of reprocessing procedures recorded in the department varied from $7.3 \%$ for bronchoscopes in the NEDD phase to $29 \%$ for bronchoscopes in the ED phase.

For each endoscope type, distributions for initial and final $\log 10$ (RLU) during both phases of the study are presented in - Fig. 1. The endoscopes in the NEDD phase seem to have initial ATP values that are higher than in the ED phase. Also, the final ATP values for the endoscopes cleaned with ED seem to be lower than those cleaned with NEDD.

Distribution of initial ATP levels found on endoscopes before manual cleaning displayed in $\mathbf{F i g} \mathbf{1}$ shows a large variability. Differences range from 1.5 logs for duodenoscopes in the NEDD phase to 4 logs for colonoscopies in the ED phase. The percentage of decrease of ATP (RLU) between initial and final 


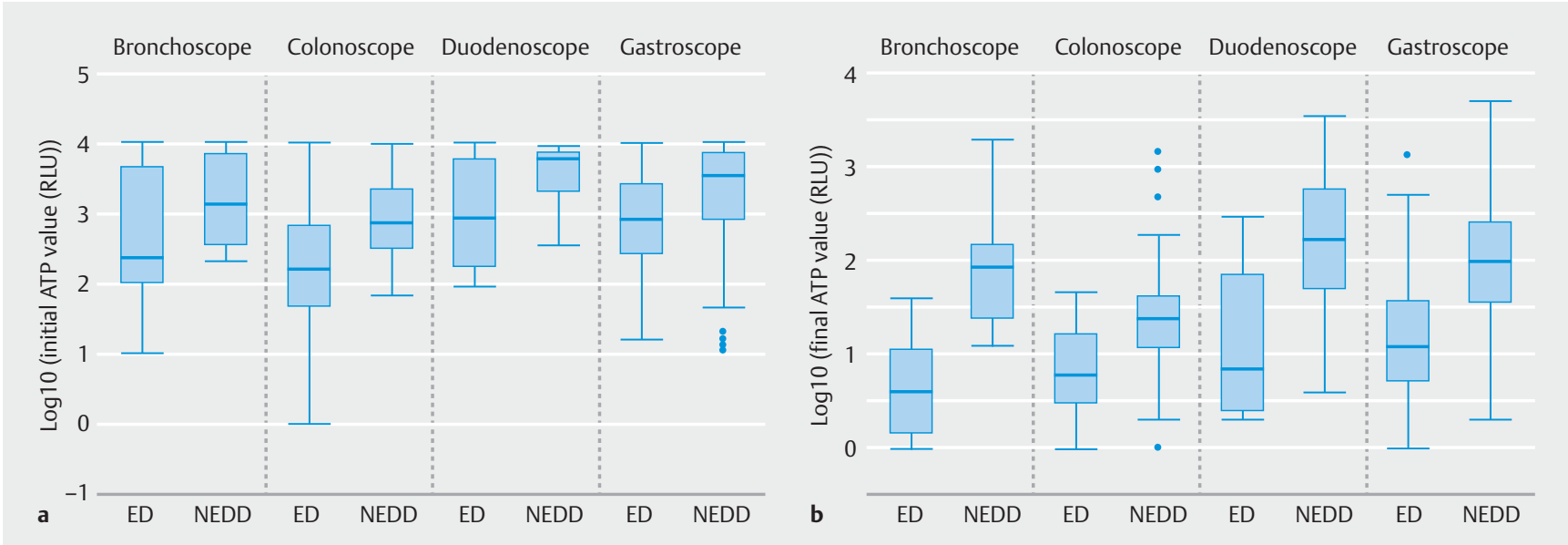

- Fig. 1 Box plots showing log10 transformation of (a) log10(initial ATP value (RLU)) and (b) log10(final ATP value (RLU)) for each detergent (NEDD and ED) and endoscope type. The central line in the box is the median, box upper and lower limits are P75 and P25 respectively and whiskers represent minimal and maximal $\log 10(R L U)$ values. Dots represent outliers, i. e. log10(RLU) values located outside a 1.5 interquartile interval from the median.

\begin{tabular}{|c|c|c|c|c|}
\hline & & Mean & Median & $P$ value of Kruskal-Wallis test \\
\hline \multirow[t]{2}{*}{ Bronchoscope } & ED & 97.68 & 99.43 & \multirow[t]{2}{*}{$0.0007^{*}$} \\
\hline & NEDD & 88.59 & 95.95 & \\
\hline \multirow[t]{2}{*}{ Colonoscope } & ED & 94.73 & 99.28 & \multirow[t]{2}{*}{$<0.0001^{*}$} \\
\hline & NEDD & 91.63 & 96.93 & \\
\hline \multirow[t]{2}{*}{ Duodenoscope } & ED & 96.68 & 97.70 & \multirow[t]{2}{*}{0.2685} \\
\hline & NEDD & 86.33 & 96.57 & \\
\hline \multirow[t]{2}{*}{ Gastroscope } & ED & 95.15 & 98.36 & \multirow[t]{2}{*}{$<0.0001^{*}$} \\
\hline & NEDD & 90.53 & 95.36 & \\
\hline
\end{tabular}

ATP levels in endoscope samples was calculated as a means to take that variability into account and assess the performance of manual cleaning procedures in both phases of the study. Mean and median values for each endoscope types are displayed in $>$ Table 2. Data distributions were not symmetrical as indicated by the discrepancies between the mean and median values observed. Kruskal-Wallis test $P$ values indicate that distributions of ATP decrease values are significantly different for ED and NEDD phases for the following endoscope types: bronchoscopes, colonoscopes and gastroscopes $(P<0.05)$ but not for duodenoscopes $(P>0.05)$. In all cases where $P$ values were below 0.05 , median ATP decrease percentages are significantly higher for ED compared to NEDD, respectively $99.43 \%$ and $95.95 \%$ for bronchoscopes, $99.28 \%$ and $96.93 \%$ for colonoscopies and $98.36 \%$ and $95.36 \%$ for gastroscopes. Although they cannot be directly compared with a statistical test, mean ATP decreases were also higher with ED than with NEDD.

To determine whether the detergent influenced the ability of the manual cleaning procedure to bring the endoscope repeatedly below a certain level of residual soiling measured with ATP, different RLU thresholds were used: 50, 100, 150, and 200 RLUs. The cleanliness of an endoscope was therefore considered "acceptable" if the ATP value after cleaning was less than the value of the threshold and "unacceptable" if it was greater than or equal to the threshold. Final ATP levels obtained during each phase of the study for each endoscope type were compared based on the percentage of endoscopes with acceptable cleanliness after manual cleaning. The procedures for which initial ATP values were lower than the corresponding threshold were excluded from this analysis (i.e. endoscopes were not dirty enough to be considered a cleaning challenge). 
The number of procedures analyzed for each threshold and each endoscope type are summarized in $\mathbf{T}$ Table $\mathbf{S 2}$.

The results of the threshold analysis are presented in - Fig. 2. The detergent used and endoscope cleanliness after manual cleaning were significantly associated for bronchoscopes and gastroscope for all thresholds, colonoscopes for all thresholds except $200 \mathrm{RLU}$, and duodenoscopes for thresholds 100 and 150 RLU. In all cases where a significant association was found, acceptability rate was higher for ED than for NEDD. Acceptability rates varied according to the threshold, with lower thresholds generally providing lower acceptability rates. For ED, the acceptability rate was $100 \%$ for bronchoscopes and colonoscopes (all thresholds) and ranged from 66.7\% (50 RLU) to $87.5 \%$ (100 RLU) for duodenoscopes and from 81.2\% (50 RLU) to $95.7 \%$ (200 RLU) for gastroscopes. For NEDD, acceptability rate ranged from $41.2 \%$ (50 RLU) to $82.4 \%$ (150 and 200 RLU) for bronchoscopes, from $80 \%$ (50 RLU) to $94.9 \%$ (200 RLU) for colonoscopes, from $25 \%$ (50 RLU) to $62.5 \%$ (200 RLU) for duodenoscopes and from $31.4 \%$ (50 RLU) to $66.3 \%$ (200 RLU) for gastroscopes.

\section{Discussion}

This research was focused on manual cleaning of endoscopes, an important step of the reprocessing cycle that precedes high-level disinfection, during which soil and biofilms should be physically removed. Previous studies show that, despite the fact that reprocessing was performed in compliance with the procedure provided by the manufacturer, residual soil, biofilm or living microorganisms could persist within endoscopes [13, $23-24]$. It was previously suggested that thorough and meticulous cleaning is essential to ensure effective HLD [25-26]. In this study, we investigated the role of the detergent in the outcome of routine endoscope manual cleaning in an endoscopy department. ATP quantification was used to determine the initial level of soiling of the biopsy and suction channels of endoscopes before manual cleaning and the level of cleanliness of the same channels reached after manual cleaning. This technique was chosen for several reasons. First, it was previously validated for this purpose by Alfa and colleagues [22]; second, its rapidity meant that it caused minimal interference with the routine processes [27]; and finally, its simplicity allowed us to train the cleaning staff to perform the measurement and record the data. In total, 461 procedures were analyzed, 208 in the phase where NEDD was used as cleaner and 253 in the phase where ED phase was. The detergent used for manual cleaning was the only parameter that was intentionally changed between the two phases of the study. We included 12 endoscopes in this study - four gastroscopes, four colonoscopes, two duodenoscopes and two bronchoscopes - in order to represent various types of devices that are used for distinct medical procedures and that have different features in terms of channel size and internal structure.

In our results, we first found that the initial level of soiling of endoscopes was highly variable and that was the case for all endoscopes types. Differences in initial ATP present in endoscopes up to 4 logs in ATP (RLU) (which corresponds to the su- perior limit of quantification of the Hygiena device used in this study) were found for colonoscopes, possibly due to various levels of patient readiness for the medical examination. To compensate for the variability observed in the initial ATP values, we decided to calculate an ATP decrease percentage for each of the procedures analyzed as an indicator of the performance of manual cleaning. Such an indicator takes into account both the levels of ATP before and after manual cleaning. The results showed that the distribution of ATP decrease for the ED phase was significantly different for gastroscopes, bronchoscopes, and colonoscopes than for the NEDD phase, as confirmed by the $P$ values of the Kruskal Wallis test. Manual cleaning with ED proved more consistent (less dispersion in data as indicated by the closeness between mean and median ATP decrease values) and median ATP decrease significantly higher with ED for bronchoscopes, colonoscopes and gastroscopes were observed. No conclusions could be drawn for duodenoscopes, probably due to the low number of procedures that were analyzed.

In previous studies regarding validation of the ATP technology, the concept of ATP thresholds to be reached for adequate manual cleaning was proposed and 200 RLU was deemed relevant [22]. However, ATP thresholds are strongly dependent on the sampling method and the ATP quantification device used in particular. In this study, the sampling method did not correspond to the method described by Alfa and colleagues in their validation study, nor did the ATP device used: they employed a $3 \mathrm{M}$ device whereas the ATP measurements in this study were made with a Hygiena device. Although direct correlation with other studies was not possible, four thresholds were chosen to perform acceptability analyzes - 50, 100, 150 and 200 RLU and discriminate the manual cleaning processes with each of the two detergents. Results revealed that manual cleaning with ED resulted in significantly better acceptability rates than cleaning with NEDD. That was the case for gastroscopes and bronchoscopes for all thresholds, for colonoscopes with 50, 100 and 150 RLU, and for duodenoscopes with 100 and 150 RLU. In some cases, no statistical differences were observed: for colonoscopes with a 200-RLU threshold and duodenoscopes with a 50- and 200-RLU threshold. The lack of significance observed for duodenoscopes could be attributed to the lack of statistical power of the analysis due to the low number of observations. These differences in acceptability rates indicate that performing manual cleaning with two different detergents can impact the outcome of this process. This is corroborated by the findings of another study that compared 10 detergents, used in automated washer-disinfectors to clean surrogate endoscopes soiled with bacteria and blood based on an ISO 15883-5 protocol. They highlighted that cleaning chemistry had an impact on the final cleanliness of the surrogate endoscope [28].

In this case, the enzymatic detergent was found to be superior to the non-enzymatic detergent. In the literature, there is still a controversy as to whether enzymatic or non-enzymatic detergents provide the best cleaning results for endoscopes. For example, results corroborating our findings were found in three studies that compared the ability of detergents to re- 

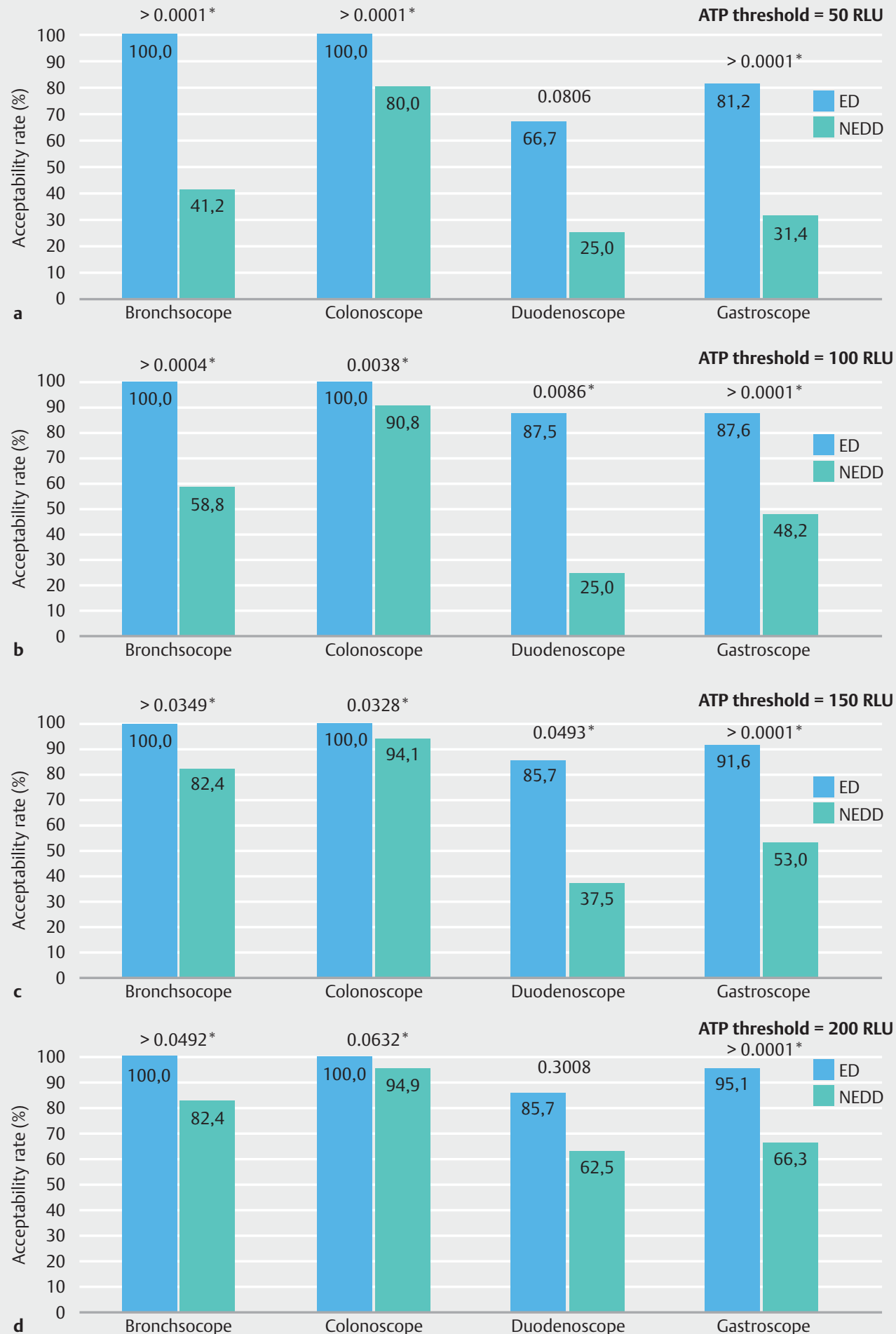

Fig. 2 Acceptablity rate of each type of endoscopes based on four post-manual cleaning ATP thresholds: (a) 50 RLU, (b) 100 RLU, (c) 150 RLU and (d) 200 RLU, during each phase of this study: ED and NEDD. Acceptability rates are indicated inside each column, just below the cap. $P$ values for the likelihood ratio test, assessing whether acceptability rates are significantly different in the two phases of this study, are indicated above the columns for each endoscope type for the four thresholds tested.

move traditional biofilms and/or soils $[25,29,30]$. However, in two other studies, enzymatic detergents were found to remove less biofilm than non-enzymatic detergents [31-32]. Of note, in both cases, the studies focused on biofilm models and soil models were not used. In addition to the influence of mechanical action, cleaning temperature, and contact time, we hypo- 
thesized that the reported differences between enzymatic and non-enzymatic cleaners mainly depend on the cleaner's formulation.

This study also revealed that some endoscopes presented a stronger cleaning challenge than others. Colonoscopes had the lowest post-manual cleaning ATP values of all endoscope types. In addition, the difference in performance of manual cleaning between the two phases of this study was also the lowest (i.e. lowest mean difference in ATP decrease between ED and NEDD and lowest difference in acceptability rate between ED and NEDD of all endoscope types). Removal of feces may be easier and less dependent on the type of detergent used. On the contrary, gastroscopes and bronchoscopes proved more discriminatory for the detergents as acceptability rates were markedly different, especially with a threshold of 50 RLU (e.g. $58.8 \%$ and $49.8 \%$ of difference in acceptability rate for bronchoscope and gastroscopes, respectively). We hypothesize that the type of soil found in these endoscopes presents a tougher challenge for cleaning and reveals differences in cleaners' performance. Despite the fact that duodenoscopes have been pointed out as difficult to adequately reprocess in the literature [33], the low number of observations for duodenoscopes in this work does not enable us to draw any conclusion for this type of endoscope.

The total number of procedures performed with the endoscopes included in this study was different in the two phases: 1708 with ED and 2263 with NEDD. During the ED phase, two endoscopes (one colonoscope and one duodenoscope) were sent to the manufacturer for repair due to mechanical issues. These two were absent from the hospital for several weeks, therefore explaining the difference in endoscope rotation between the two phases of this study.

Our work has several limitations. First, although all staff working in the endoscope reprocessing unit were fully trained, no checklist were used during this study to ensure compliance with the reprocessing procedure. Potential deviations from the standard procedures were therefore not recorded. However, one could hypothesize that, if reprocessing lapses occurred during this study, they occurred at the same rates in both phases. Second, the staff in the endoscope reprocessing department were aware of the study objectives and were not blinded. Also, ATP quantification was used as the sole method of assessing endoscope cleanliness. Including more techniques, such as test strips for protein residues or microbial culture, could have provided additional insights. However, culture was not implemented in this study because the amount of time required to proceed to aseptic sampling was not compatible with routine processes at play. Because the sampling procedure had to be quick, only the biopsy and suction channels of endoscopes were sampled in this study. Therefore, the results of this study are not necessarily transposable to the cleanliness of other, smaller endoscope channels that cannot be brushed, e.g. airwater channel.

\section{Conclusion}

This study shows that the detergent used to perform manual cleaning of endoscopes has a major impact on the level of cleanliness of endoscopes prior to disinfection in AER. In this case, the enzymatic detergent was found superior to the nonenzymatic one, leaving significantly less measurable ATP in the endoscopes at the end of the manual cleaning step. The conclusions of our study are not necessarily generalizable for several reasons. First, in further studies, other types of detergents (e.g. non-enzymatic and non-biocidal) like slight alkaline detergents should be investigated in comparison. Second, experiments were only performed at a single hospital site. Multicenter deployment of this protocol could be the object of a separate study to validate our results in other healthcare facilities. Third, only Olympus endoscopes were included in this study. A future larger study could include endoscopes from other manufacturers. Finally Including cystoscopes or ureteroscopes in a future study would be of interest, especially because they enter sterile cavities and are classified as critical devices.

\section{Acknowledgements}

The authors thank Dr. Guy Heynen, Prof. Michel Goldman and Prof. Michel Delmée who kindly reviewed this manuscript prior to submission. Statistical analyses were performed by SMCS, the statistical platform of the Université Catholique de Louvain.

\section{Competing interests}

OneLIFE provided financial support for the ATP tests and for one extra staff member on the endoscope cleaning and disinfection team to cope with the extra work generated by the study. TV is R\&D manager at OneLIFE S.A., the company that markets the enzymatic detergent (ED) used in this study.

\section{References}

[1] Rutala WA, Weber D]. HICPAC. CDC Guidelines for Disinfection and Sterilization in Healthcare Facilities. 2008: 1-27

[2] Owings MF, Kozak LJ. Ambulatory and inpatient procedures in the United States, 1996. Vital Heal Stat 1998; 13: 1-119

[3] Spaulding E. Chemical disinfection of medical and surgical materials. In: Lawrence C, Block S (Hrsg.) Disinfection, Sterilization, and Preservation. Philadelphia: Lea \& Febriger; 1968: 517- 531

[4] Banerjee S, Shen B, Nelson DB et al. Infection control during GI endoscopy. Gastrointest Endosc 2008; 67: 781 - 790

[5] Beilenhoff U, Neumann CS, Rey JF et al. ESGE-ESGENA guideline: Cleaning and disinfection in gastrointestinal endoscopy: Update 2008. Endoscopy 2008; 40: 939-957

[6] Joshi SG. Acinetobacter baumannii : An emerging pathogenic threat to public health. World J Clin Infect Dis 2013; 3: 25-36

[7] Rutala WA, Weber D]. New developments in reprocessing semicritical items. Am J Infect Control 2013; 41: 60-66

[8] Bradford BD, Seiberling KA, Park FE et al. Disinfection of rigid nasal endoscopes following in vitro contamination with Staphylococcus aureus, Streptococcus pneumoniae, Pseudomonas aeruginosa, and 
Haemophilus influenzae. JAMA Otolaryngol Head Neck Surg 2013; 139: $574-578$

[9] Kovaleva J, Peters FTM, van der Mei HC et al. Transmission of infection by flexible gastrointestinal endoscopy and bronchoscopy. Clin Microbiol Rev 2013; 26: 231 - 254

[10] Bajolet O, Ciocan D, Vallet C et al. Gastroscopy-associated transmission of extended-spectrum beta-lactamase-producing Pseudomonas aeruginosa. J Hosp Infect 2013; 83: $341-343$

[11] Kola A, Piening B, Pape U-F et al. An outbreak of carbapenem-resistant OXA-48 - producing Klebsiella pneumonia associated to duodenoscopy. Antimicrob Resist Infect Control 2015; 4: 8-12

[12] Epstein L, Hunter JC, Arwady MA et al. New Delhi metallo- $\beta$-lactamase-producing carbapenem-resistant Escherichia coli associated with exposure to duodenoscopes. Jama 2014; 312: 1447-1455

[13] Neves MS, da Silva MG, Ventura GM et al. Effectiveness of current disinfection procedures against biofilm on contaminated Gl endoscopes. Gastrointest Endosc 2016; 83: 944 - 953

[14] Alfa MJ, Fatima I, Olson N. The adenosine triphosphate test is a rapid and reliable audit tool to assess manual cleaning adequacy of flexible endoscope channels. Am J Infect Control 2013: 41

[15] Alfa MJ, Olson N. Simulated-use validation of a sponge ATP method for determining the adequacy of manual cleaning of endoscope channels. BMC Res Notes 2016; 9: 258-264

[16] Fushimi R, Takashina M, Yoshikawa $\mathrm{H}$ et al. Comparison of adenosine triphosphate, microbiological load, and residual protein as indicators for assessing the cleanliness of flexible gastrointestinal endoscopes. Am J Infect Control 2013; 41: 161-164

[17] Qiu L, Zhou Z, Liu Q et al. Investigating the failure of repeated standard cleaning and disinfection of a Pseudomonas aeruginosa-infected pancreatic and biliary endoscope. Am J Infect Control 2015; 43: e43e46

[18] Ofstead CL, Westzler HP, Doyle EM et al. Persistent contamination on colonoscopes and gastroscopes detected by biologic cultures and rapid indicators despite reprocessing performed in accordance with guidelines. Am J Infect Control 2015; 43: 794-801

[19] Ofstead CL, Wetzler HP, Heymann OL et al. Longitudinal assessment of reprocessing effectiveness for colonoscopes and gastroscopes: Results of visual inspections, biochemical markers, and microbial cultures. Am J Infect Control 2017; 45: e26 - e33

[20] Ofstead CL, Wetzler HP, Snyder AK et al. Endoscope reprocessing methods. Gastroenterol Nurs 2010; 33: 304-311
[21] Hervé R, Keevil CW. Current limitations about the cleaning of luminal endoscopes. J Hosp Infect 2013; 83: 22-29

[22] Alfa M], Fatima I, Olson N. Validation of adenosine triphosphate to audit manual cleaning of flexible endoscope channels. Am J Infect Control 2013; 41: 245-248

[23] Ofstead CL, Wetzler HP, Doyle EM et al. Persistent contamination on colonoscopes and gastroscopes detected by biologic cultures and rapid indicators despite reprocessing performed in accordance with guidelines. Am J Infect Control 2015; 43: 794-801

[24] Aumeran C, Thibert E, Chapelle F et al. Assessment on experimental bacterial biofilms and in clinical practice of the efficacy of sampling solutions for microbiological testing of endoscopes. J Clin Microbiol 2012; 50: $938-942$

[25] da Costa LucianoC, Olson N, Tipple AFV et al. Evaluation of the ability of different detergents and disinfectants to remove and kill organisms in traditional biofilm. Am J Infect Control 2016; 44: e243 - e249

[26] Martiny H, Floss H, Zühlsdorf B. The importance of cleaning for the overall results of processing endoscopes. J Hosp Infect 2004; 56: 16 22

[27] Gillespie E, Sievert W, Swan M et al. Adenosine triphosphate bioluminescence to validate decontamination of endoscopes. J Hosp Infect 2017; 97: 353-356

[28] Zühlsdorf B, Floss H, Martiny H. Efficacy of 10 different cleaning processes in a washer-disinfector for flexible endoscopes. J Hosp Infect 2004; 56: $305-311$

[29] Hadi R, Vickery K, Deva A et al. Biofilm removal by medical device cleaners: comparison of two bioreactor detection assays. J Hosp Infect 2010; 74: $160-167$

[30] Stiefel P, Mauerhofer S, Schneider J et al. Enzymes enhance biofilm removal efficiency of cleaners. Antimicrob Agents Chemother 2016; 60: $3647-3652$

[31] Ren W, Sheng X, Huang X et al. Evaluation of detergents and contact time on biofilm removal from flexible endoscopes. Am J Infect Control 2013; 41: 1-4

[32] Fang Y, Shen Z, Li L et al. A study of the efficacy of bacterial biofilm cleanout for gastrointestinal endoscopes. World J Gastroenterol 2010; 16: $1019-1024$

[33] Humphries RM, McDonnell G. Superbugs on duodenoscopes: The challenge of cleaning and disinfection of reusable devices. J Clin Microbiol 2015; 53: 3118-3125 
- Table S1 S Endoscopes included in this study.

\begin{tabular}{|l|l|l|l|l|}
\hline Endoscope type & Endoscope brand & Internal endoscope code & Serial number & Entry in service \\
\hline Gastroscope & Olympus & 106 & 2400451 & May 2014 \\
\hline Gastroscope & Olympus & 204 & 2415525 & May 2014 \\
\hline Gastroscope & Olympus & 211 & 2519086 & May 2015 \\
\hline Gastroscope & Olympus & 203 & 2205733 & April 2013 \\
\hline Colonoscope & Olympus & 210 & 2500478 & May 2015 \\
\hline Colonoscope & Olympus & 212 & 2510896 & May 2015 \\
\hline Colonoscope & Olympus & 207 & 2410586 & 2400215 \\
\hline Colonoscope & Olympus & 209 & 2801843 & May 2014 \\
\hline Bronchoscope & Olympus & 405 & 2102250 & May 2014 \\
\hline Bronchoscope & Olympus & 406 & 2405851 & May 2013 \\
\hline Duodenoscope & Olympus & 301 & 2303650 & May 2015 \\
\hline Duodenoscope & Olympus & 107 & Jan 2013 \\
\hline
\end{tabular}

- Table S2 S Number of procedures included in the ATP threshold analysis for each endoscope type and each of the two phases of the study.

\begin{tabular}{|c|c|c|c|c|c|}
\hline & & \multicolumn{4}{|c|}{ ATP thresholds (RLU) } \\
\hline & & 50 & 100 & 150 & 200 \\
\hline \multirow[t]{2}{*}{ Bronchoscope } & ED & 23 & 19 & 17 & 14 \\
\hline & NEDD & 17 & 17 & 17 & 17 \\
\hline \multirow[t]{2}{*}{ Colonoscope } & ED & 68 & 57 & 47 & 41 \\
\hline & NEDD & 90 & 87 & 84 & 78 \\
\hline \multirow[t]{2}{*}{ Duodenoscope } & ED & 9 & 8 & 7 & 7 \\
\hline & NEDD & 8 & 8 & 8 & 8 \\
\hline \multirow[t]{2}{*}{ Gastroscope } & ED & 122 & 113 & 106 & 102 \\
\hline & NEDD & 86 & 83 & 83 & 83 \\
\hline
\end{tabular}

